\title{
Undifferenced GPS Ambiguity Resolution using the Decoupled Clock Model and Ambiguity Datum Fixing
}

\author{
Paul Collins \\ York University, Toronto, Ontario, Canada \\ Natural Resources Canada, Ottawa, Ontario, Canada \\ Sunil Bisnath \\ York University, Toronto, Ontario, Canada \\ François Lahaye and Pierre Héroux \\ Natural Resources Canada, Ottawa, Ontario, Canada
}

\begin{abstract}
This paper describes a method of processing Global Positioning System (GPS) observations to achieve undifferenced ambiguity resolution. Dual-frequency carrier phase and pseudorange measurements are processed by specifying separate oscillator parameters for the carrier phase and pseudorange measurements. Carrier phase estimates of the oscillator errors are arbitrarily biased with respect to the pseudorange estimates, and ambiguity parameters are constrained to be integer-valued. A network solution is necessary to compute the satellite code and phase clocks required as fixed parameters in single-user Precise Point Positioning (PPP) solutions. In the PPP results presented here, the LAMBDA method was used to optimally resolve ambiguities at each epoch. Only a small improvement in position error is gained with 5minute static processing over 24 hours. However, there is a significant reduction in convergence time, and with $30-\mathrm{sec}$ static processing after 60 minutes, $90 \%$ of solutions approach $2 \mathrm{~cm}$ horizontal error, compared to $10 \mathrm{~cm}$ for standard PPP.
\end{abstract}




\section{INTRODUCTION}

A key issue in Global Navigation Satellite Systems (GNSS), including the Global Positioning System (GPS), is the isolation and estimation of the integer ambiguity of the carrier phase measurements. The carrier phase signals of any GNSS are approximately two orders of magnitude more precise than the primary pseudorange signals the systems provide. However, measurements of the carrier phases are ambiguous relative to those of the pseudoranges by an unknown number of integer cycles [20]. Resolution of these ambiguities converts the carrier phases into pseudorange measurements, with measurement noise at the centimeter-to-millimeterlevel compared to the decimeter-to-meter-level of the direct pseudoranges. The improvement in measurement precision is directly carried into the parameters estimated from the measurements.

Until recently, ambiguities were only resolved as integers in so-called double difference processing whereby dual-pairs of measurements (made for example from two receivers to the same two satellites) are differenced to produce one measurement [4]. The differencing is carried out primarily to remove common transmitter and receiver biases contained in the measurements. These biases consist primarily of the oscillator-induced time delays of both the satellite and the receiver. The existence of these timing biases is why the measurements are 'pseudoranges' and not just 'ranges'.

The primary disadvantage of double differencing is the requirement to have at least two receivers, even for a single user who only requires his or her own location. This essentially turns point positioning into baseline, or relative, positioning and can involve considerable processing 
overheads for the user. The technique can also be very limited in baseline length if such error sources as orbits and ionosphere are required to cancel out.

As an alternative to double differencing, it is possible to process undifferenced measurements and estimate the biases explicitly. It can be shown that the two solutions are mathematically identical under certain circumstances [27]. At the same time, it is not possible to uniquely isolate the integer nature of the ambiguities due to their exact linear correlation with the time delays of the oscillators and other hardware. The higher precision of the carrier phase can still be accessed by estimating a random constant bias in place of the ambiguity. However, accurately estimating such a parameter requires an extended convergence period.

The processing of undifferenced pseudorange and carrier phase observables from a single receiver is referred to as Precise Point Positioning (PPP) $[28,16]$. PPP returns in effect to the first principles of GPS, where the focus is again placed on a single receiver, and where the userrequired corrections are satellite-based only. The main challenge with PPP is the significant convergence period required due to the necessity of estimating real-valued ambiguities. This convergence period is the most significant factor limiting wider adoption of PPP [1]. If the ambiguities could be isolated and estimated as integer values then, in principle, their integer nature represents more information that can be exploited to accelerate convergence.

Accordingly, integer ambiguity resolution of undifferenced carrier phase observables has been an elusive goal, and the subject of much research in GPS processing, largely since the advent of the PPP method. Some recent advances in isolating integer ambiguities have been made with 
techniques that use single differences [13] and undifferenced observables $[17,18]$. However, it is not clear that all aspects of the problem have been addressed, particularly with respect to timevarying code biases that are not explicitly accommodated by these techniques.

For our purposes, the term 'code biases' refers to unmodeled common-mode errors of the pseudoranges, usually considered to be hardware or local environment delays, that are either constant, or believed to vary in a band-limited, quasi-random, manner. There appears to be general acceptance in the area of precise time transfer, that these biases are the cause of the socalled 'day-boundary clock jumps' highlighted by the time scale of the International GNSS Service (IGS) (see, e.g., [19], [11] and [15]). The term 'phase biases' refers to analogous delays of the carrier phase measurements.

This paper will show that the limiting factor in ambiguity resolution using undifferenced GPS observables is the presence of both code and phase biases in the estimates of the ambiguities. As parameterized in the 'standard model' of undifferenced ionosphere-free pseudoranges and carrier phases, the datum for the station and satellite clock parameters is provided by the pseudoranges. The consequence of this is that the estimated ambiguities contain the time-constant portions of both code and phase biases. To address the limitations and problems of the standard model, a new GPS observation model is presented called the 'decoupled clock model'. This model rigorously accommodates any synchronization biases due to hardware delays (constant or timevarying) that may occur between common-frequency observables and facilitates the estimation of integer ambiguities without explicit differencing. 


\section{STANDARD OBSERVATION MODEL}

The standard GPS dual-frequency pseudorange (code) and carrier phase (phase) observation equations are typically written in the form:

$$
\begin{aligned}
P_{1} & =\rho+T+I+c\left(d t^{r}-d t^{s}\right)+b_{P 1}^{r}-b_{P 1}^{s}+\varepsilon_{P 1} \\
P_{2} & =\rho+T+q^{2} I+c\left(d t^{r}-d t^{s}\right)+b_{P 2}^{r}-b_{P 2}^{s}+\varepsilon_{P 2} \\
\lambda_{1}\left(\Phi_{1}+N_{1}\right) & = \\
L_{1} & =\rho+T-I+c\left(d t^{r}-d t^{s}\right)+b_{L 1}^{r}-b_{L 1}^{s}+\varepsilon_{L 1} \\
\lambda_{2}\left(\Phi_{2}+N_{2}\right) & = \\
L_{2} & =\rho+T-q^{2} I+c\left(d t^{r}-d t^{s}\right)+b_{L 2}^{r}-b_{L 2}^{s}+\varepsilon_{L 2}
\end{aligned}
$$

where $P_{\mathrm{i}}$ is a pseudorange measurement made at frequency $\mathrm{i}$ and $\Phi_{\mathrm{i}}$ is a carrier phase measurement made at frequency i. We write the integer ambiguity $N_{\mathrm{i}}$ on the left side to show how it converts the ambiguous phase measurement $\Phi_{\mathrm{i}}$ into a precise pseudorange $L_{\mathrm{i}}$. The factor $q$ represents the ratio of the primary and secondary GPS frequencies, $c$ is the vacuum speed of light and $\lambda_{\mathrm{i}}$ is the frequency-dependent wavelength of the carrier phase measurements. Of the geometric parameters, $\rho$ represents the geometric range between transmitter and receiver antennas, $T$ is the range delay caused by signal propagation through the lower atmosphere (predominantly the Troposphere), and $I$ is the range delay and apparent phase advance on the

primary frequency caused by signal propagation through the upper atmosphere (predominantly the Ionosphere). The remaining, non-geometric, parameters are the oscillator or 'clock' errors for both the transmitter and receiver ( $d t^{s}$ and $d t^{r}$, respectively), and common-oscillator hardware 
biases $\left(b_{*}^{*}\right)$ for each observation. Unmodeled random or quasi-random errors are represented by $\varepsilon_{*}$.

The usual practice when processing dual-frequency measurements is to take advantage of the frequency-dependence of the ionospheric delay (to first order) and linearly combine the pseudorange and carrier phase observables to produce ionosphere-free observables:

$$
\begin{aligned}
& P_{3}=\rho+T+c\left(d t^{r}-d t^{s}\right)+b_{P 3}^{r}-b_{P 3}^{s}+\varepsilon_{P 3} \\
& L_{3}=\rho+T+c\left(d t^{r}-d t^{s}\right)+b_{L 3}^{r}-b_{L 3}^{s}-\lambda_{3} N_{3}+\varepsilon_{L 3}
\end{aligned}
$$

where:

$$
P_{3}=\frac{\left(77^{2} P_{1}-60^{2} P_{2}\right)}{\left(77^{2}-60^{2}\right)}, L_{3}=\frac{\left(77^{2} L_{1}-60^{2} L_{2}\right)}{\left(77^{2}-60^{2}\right)}
$$

and the ionosphere-free ambiguity combination $N_{3}=77 N_{1}-60 N_{2}$ is placed on the right-hand side to indicate that it is now treated as a parameter to be estimated from the data.

As they stand, these equations are over-parameterized, and any system of normal equations derived from them for the purposes of a least squares solution will be singular. There are two causes of deficiency. The first is that the clock errors are inherently differenced and cannot be uniquely separated. This is overcome in a network solution by fixing one of the station clocks, and in a single-receiver solution by fixing the satellite clocks. The remaining singularity is due to the presence of the hardware biases and their identical functional behavior with the associated 
clock parameters. Both these types of parameter represent common-mode time delays and, having constant partial derivatives, are not uniquely separable.

To explicitly deal with this singularity, equivalent equations can be written with clock and code bias parameters combined. At the same time, due to the uniquely ambiguous nature of the carrier phase, the combined clock and bias parameter can be carried over to the phase observable:

$$
\begin{aligned}
& P_{3}=\rho+T+c\left(d t_{P 3}^{r}-d t_{P 3}^{s}\right)+\varepsilon_{P 3} \\
& L_{3}=\rho+T+c\left(d t_{P 3}^{r}-d t_{P 3}^{s}\right)+A_{P 3}+\varepsilon_{L 3}
\end{aligned}
$$

where $c \cdot d t_{P 3}^{*}=c \cdot d t^{*}+b_{P 3}^{*}$, and compensating code biases plus the phase biases and the ambiguity are combined into one parameter:

$$
A_{P 3}=b_{L 3}^{r}-b_{P 3}^{r}-b_{L 3}^{s}+b_{P 3}^{s}-\lambda_{3} N_{3}
$$

which is sometimes referred to as the 'float' or 'real-valued' ambiguity because it is not integer valued. The justification for grouping these parameters is that they are all functionally identical (constant measurement model partial derivatives) and as a random bias, the integer ambiguity cannot be independently predicted a-priori.

These equations will be referred to as the standard model for dual-frequency undifferenced processing, despite being in what may appear to be a non-standard form. These equations rigorously represent the combined effect of common-mode code-biases, common mode phasebiases, common-observation clocks and random ambiguities. The net effect is that the ambiguity 
parameter of the standard model contains both phase and code time-constant biases. Justification for using this formulation is provided in the next section.

\section{DAY-BOUNDARY CLOCK JUMPS}

Up to this point we have assumed that the code biases are constant over time. However, this is largely accepted as untrue, especially by precise timing users of GPS (see, e.g., [10]), but the detailed impact seems not to be properly understood. Significant work has been done to investigate the impact of time-varying code biases and their apparent manifestation as the dayboundary clock jumps identified in the IGS Time Scale [19]. We will show now that these jumps are caused by time-varying code-biases and exist precisely because code and phase observations are mixed together with common oscillator parameters in the standard model.

Figure 1 presents three time-series representing the relative clock performance over seven days of two Hydrogen Maser oscillators located at Boulder, Colorado, U.S.A. (AMC2) and Yellowknife, Northwest Territories, Canada (YELL). These oscillators provide the frequency input to the geodetic-GPS receivers located at the two sites. A common slope has been subtracted from all the time series to remove the relative offset and drift. The initial solution to consider is the IGS Rapid solution. This is a set of daily solutions resulting from the combination of several independent batch solutions processed using the standard model with

backward processing and parameter smoothing. In comparison, there are two sequential solutions computed with neither backward processing nor parameter smoothing. These consist 
of a straightforward code-and-phase standard model solution and a code-only solution. For these two solutions the clocks were estimated as white-noise parameters with no a-priori weights.

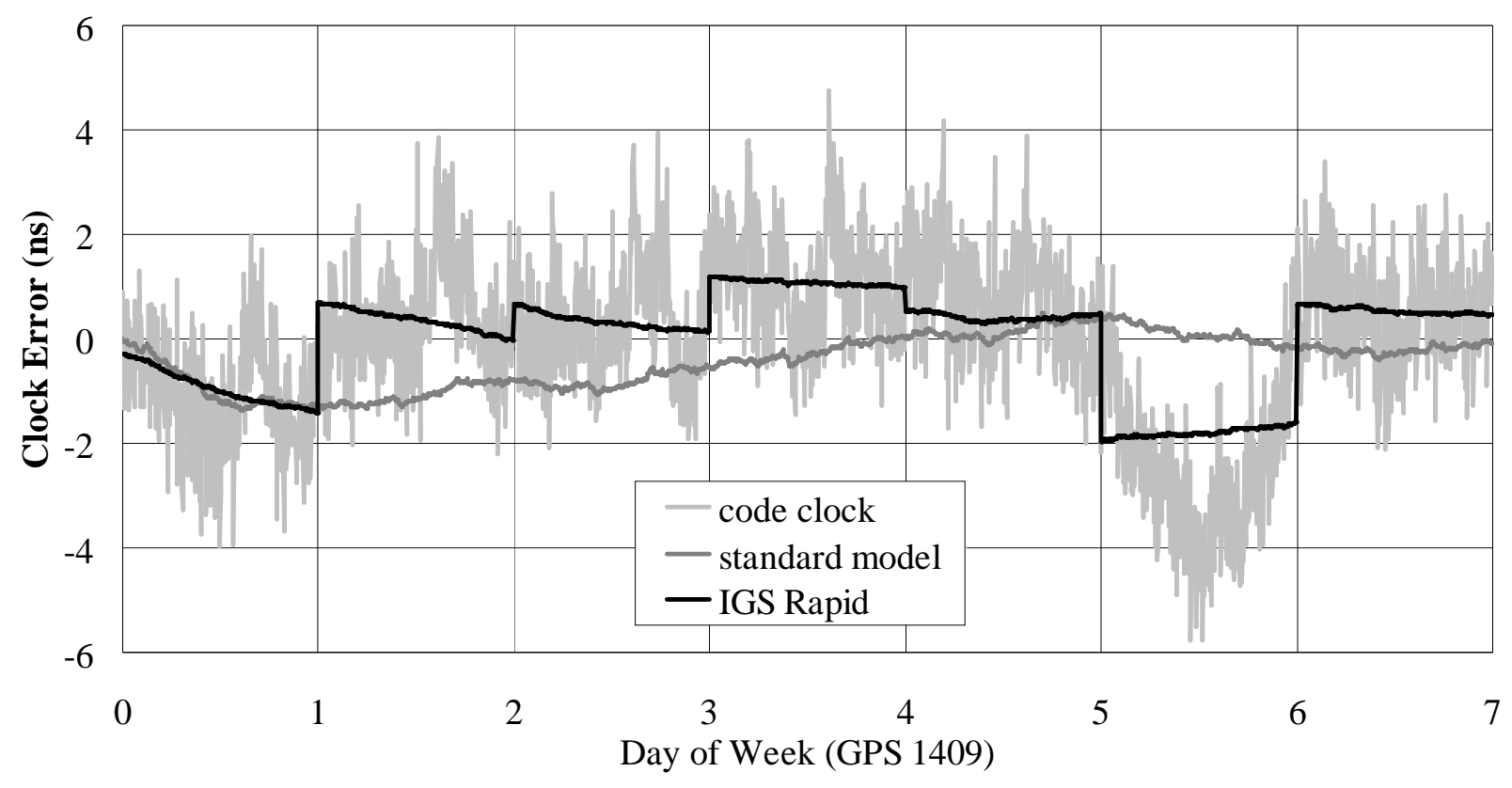

Figure 1. YELL-AMC2 clock error estimates. (Jan 7th-Jan 13th, 2007; common linear fit removed).

The day boundary jumps are clearly visible in the IGS Rapid solution. What is also clear is just how closely these jumps coincide with the underlying changes experienced by the code-only solution. As expected, the code-only solution contains significantly more noise, but what might not be expected is that there are significant trends in the solution that are not characteristic of hydrogen maser clock and geodetic receiver operation (for example, the half-sinusoidal signal during day 5).

The code-and-phase standard model solution is free of the day-boundary jumps due to the continuous nature of the processing, but the change in the underlying code also influences the solution. A clear drift is visible between day-boundaries 1 and 5 as the solution tries to realign 
itself with the code datum (represented by the code-only solution). These results emphasize that batch processing of the standard model accurately captures the frequency error of the underlying clocks, but not the relative offset; and that sequential processing of the standard model captures neither the frequency nor the offset to a level of accuracy corresponding to the phase observations.

To continue this portion of the investigation, it is relevant to ask what happens to the significant variations evident in the code-only solution compared to the code-and-phase solution. After all, these solutions processed the same code observables and yet show significantly different characteristics. The answer lies in the code residuals. Figure 2 shows the ionosphere-free code observation residuals from the last three days of the sequential standard model solution for station YELL. The half-sinusoidal trend evident in the code-only solution is clearly visible. What is most interesting however, is that these residuals are not epoch zero-mean. The epoch average of the residuals are also plotted on Figure 2 and abstracted along with those for station AMC2 in Figure 3. The lack of a half-sinusoidal trend for AMC2 indicates that station YELL is the source of this signal. More importantly however, the code residuals of neither station are zero-mean. We might expect that the combined code and phase residuals, properly weighted, are zero-mean, but the two are treated as physically distinct observables and are processed as mathematically uncorrelated (diagonal weight matrix). If the observation model is correct, then both observable types should exhibit zero-mean behaviour in their respective residuals. Therefore, our interpretation of this effect is that there are apparent common-mode signals that exist on the pseudorange measurements, but not the carrier phases. Even though these 'code- 
biases' can have a quasi-random signature at a well behaved station such as AMC2, they represent unique signals that should be rigorously modeled.

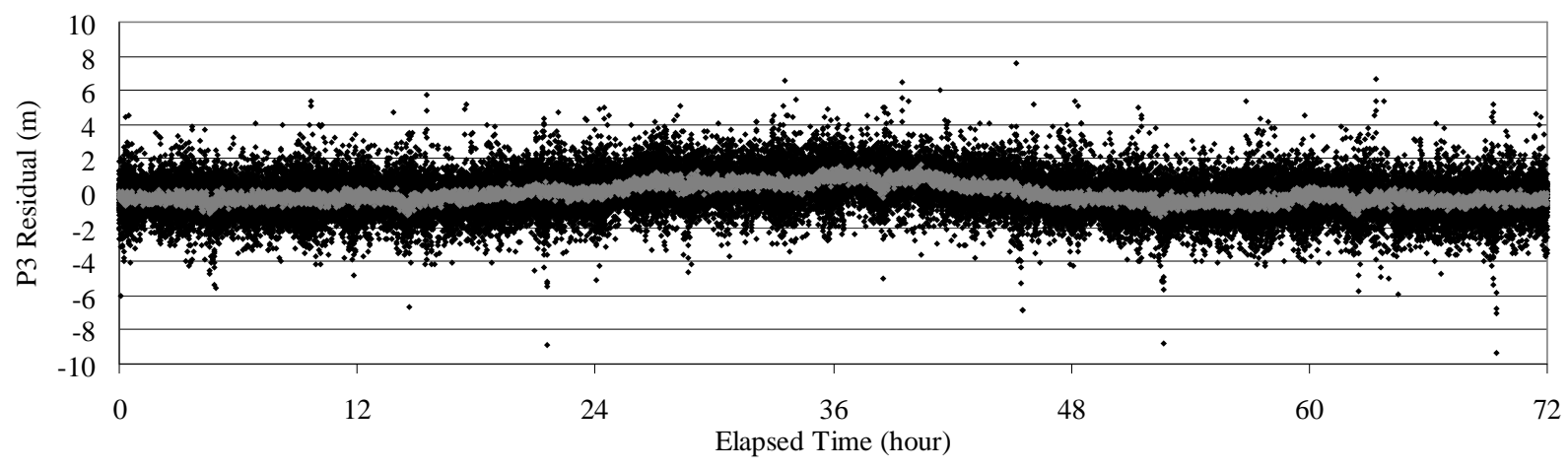

Figure 2. YELL 3-day code individual and epoch average residuals.

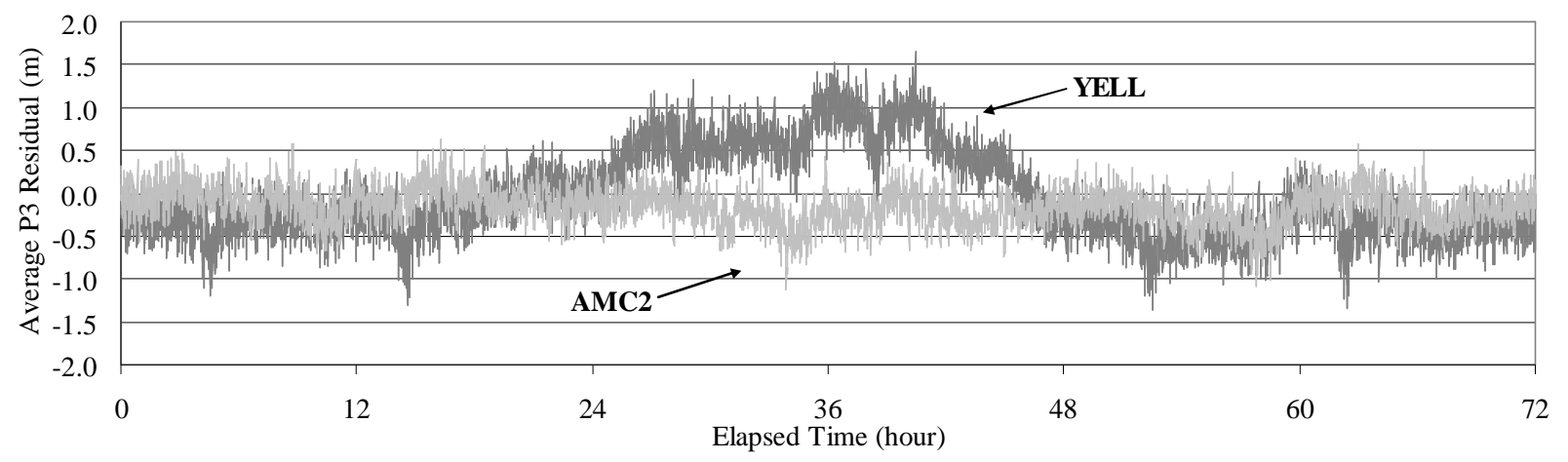

Figure 3. YELL and AMC2 3-day epoch average code residuals.

It should be clear from this discussion that the standard model of undifferenced ionosphere-free observables is sub-optimal, in that the estimated ambiguities contain the constant code as well as phase biases. Should the code or phase biases also vary over time, then the standard model is even less optimal, and any such variations must be accommodated by the other estimated parameters, such as the clocks and ambiguities, and the residuals. We will now discuss how these effects may be rigorously accounted for, and how that in turn isolates undifferenced ambiguities as integer-valued parameters. 


\section{DECOUPLED CLOCK MODEL}

We have seen from some fairly straightforward processing of the standard observation model that the average code bias contaminates the estimated phase ambiguities, and that in fact the code biases should not be considered to be constant or even narrowly varying. What are usually termed 'code-biases' represent significant and unique signals that should be rigorously modeled. We now turn to how that might be achieved and how that in turn facilitates direct estimation of integer ambiguity parameters.

Perhaps the most straightforward interpretation of Figure 3 is that the code observables are not apparently synchronized to the phase observables at the level of precision of the phases. At the same time we should recall that there is no functional difference between the code-bias signal and the underlying oscillator. Hence, the first step to take is to accept that we cannot in general separate the code biases from the underlying oscillator. Treating the phase biases analogously implies a decoupling of the clock parameters of the code and phase observables. Therefore we will write equivalent ionosphere-free observation equations with separate code and phase satellite and station clock parameters:

$$
\begin{aligned}
& P_{3}=\rho+T+c\left(d t_{P 3}^{r} \quad-d t_{P 3}^{s} \quad\right) \quad+\varepsilon_{P 3} \\
& L_{3}=\rho+T+c\left(\quad d t_{L 3}^{r}-d t_{L 3}^{s}\right)-\lambda_{3} N_{3}+\varepsilon_{L 3}
\end{aligned}
$$

These equations again represent a singular system, and with integer ambiguities, their formal solution is an example of rank-defect integer least-squares $[22,24]$. From an analytical point-ofview, the primary implication of formulating separate code and phase clock parameters is that 
the datum provided by the pseudoranges has been removed from the carrier phases and therefore an alternative must be provided. We will do so in a way that also explicitly isolates the ambiguities as integers — with ambiguity datums.

\section{AMBIGUITY DATUM FIXING}

The effect of decoupling the code and phase clock parameters is to make the system of equations singular again. However, the singular nature of the clock differences on the code and phases is now separate, and on the phases is matched by the singular nature of the ambiguities as well. The lack of a unique separation between the phase clocks and the ambiguities can be exploited to provide enough constraints so that a least-squares solution can be solved from the first epoch of processing.

Even though it is the decoupling of the clock parameters that has created a singular solution, the overall singularity lies as much with the ambiguities as with the phase clocks. If the ambiguity parameters could be removed, the carrier phases would be no more singular than the pseudoranges. Hence, fixing a subset of ambiguities provides a replacement datum for the phase clocks. Consequently, all that is required to provide a minimum-constraint least-squares solution when using the decoupled clock model is to arbitrarily fix one ambiguity associated with each estimated phase clock (less one in a network solution). By this process, the inherent ambiguity in the phase measurements is moved to the phase clock parameter and does not impact the other parameters. 
The concept of fixing ambiguities to provide redundancy for a least-squares solution of undifferenced phase observations is not entirely new. For example, the base-station-basesatellite concept was introduced by [14] for precisely that purpose. The practical impact of fixing ambiguities in this way ensures that any phase biases are associated with the underlying oscillator error to form a 'phase clock' parameter. At the same time, fixing the datum ambiguities to integer values ensures the remaining ambiguities are formally integer in nature. The impact can be considered as an implicit differencing effect, an example of which is given by [5] along with the relationship between datum fixing and the base-station-base-satellite method. More generally, the choice of ambiguities creates a spanning tree over the observation graph, an example of which is given in [6]. The concept of ambiguity datum fixing plays a role in undifferenced processing corresponding to, and likely equivalent to that of, reference satellite and baseline choice in double-difference processing.

\section{EXTENDED MODEL FOR GPS L1/L2 PROCESSING}

Straightforward processing of ionosphere-free observables with the decoupled clock model and ambiguity datum fixing provides solutions where the estimated ambiguities are formally integer valued. However, for GPS $\mathrm{L}_{1} / \mathrm{L}_{2}$ processing explicit ambiguity fixing is not practicable because the ionosphere-free wavelength is too short $\left(\lambda_{3} \sim 6 \mathrm{~mm}\right)$ relative to the phase noise. In addition, the phase clock estimates will drift due to the inability to maintain datum continuity. Datum continuity becomes an issue when a datum ambiguity drops out of the solution and a new one 
must be chosen in its place. Strict datum continuity can only be maintained when the new datum ambiguity has converged to, or been fixed at, its correct integer value.

For GPS $\mathrm{L}_{1} / \mathrm{L}_{2}$ processing therefore, the widelane-phase/narrowlane-code combination is used to first fix the widelane ambiguity (wavelength $\sim 86 \mathrm{~cm}$ ), which in turn amplifies the ionosphere-free wavelength to that of the narrowlane $(\sim 11 \mathrm{~cm})$. This extended model is mandatory for integer ambiguity resolution with GPS $\mathrm{L}_{1} / \mathrm{L}_{2}$ processing. The necessity for this step is determined by the relationship between the frequencies of the observables. For $\mathrm{L}_{2} / \mathrm{L}_{5}$ processing with GPS-III for example, an extended model will not provide any benefit, because the ionosphere-free combination wavelength is already at $12.5 \mathrm{~cm}$ and widelane substitution has no effect [5].

The widelane-phase/narrowlane-code observable (also known as the Melbourne-Wübbena combination) is defined as:

$$
A_{4}=L_{4}-P_{6}=b_{A 4}^{r}-b_{A 4}^{s}-\lambda_{4} N_{4}+\varepsilon_{A 4}
$$

where $L_{4}=\left(77 L_{1}-60 L_{2}\right) / 17$ is the widelane phase combination and $P_{6}=\left(77 P_{1}+60 P_{2}\right) / 137$ is the narrowlane code combination. It is usual when using this combination to consider the station and satellite biases as constant, however this cannot generally be justified due to the timevarying code biases. Therefore, the decoupled clock model will be invoked and these biases will be treated as time-varying. At the same time ambiguity and network datum fixing is applied to introduce redundancy into the system. As a third step, this observable is processed simultaneously with $\mathrm{P}_{3}$ and $\mathrm{L}_{3}$ to provide a homogeneous system of equations. In theory this 
step should only be undertaken with rigorous error propagation from the raw $\mathrm{P}_{1}, \mathrm{P}_{2}, \mathrm{~L}_{1}$ and $\mathrm{L}_{2}$ observables, however the relative correlation between $\mathrm{A}_{4}$ and $\mathrm{P}_{3}$ and $\mathrm{L}_{3}$ is theoretically small enough that it is possible to ignore it in practice [5].

There are two benefits to processing the widelane/narrowlane combination simultaneously with the ionosphere-free observables. The first is that the station and satellite widelane 'biases' can be treated as non-constant clock-like parameters identical to the code and phase clocks. Additionally, the least-squares system is less vulnerable to incorrectly fixed widelane ambiguities biasing the solution. Residual testing can be used to restart estimation of both the widelane and ionosphere-free ambiguities if bad fixes on either observable are suspected. Both effects provide for a more robust solution, with as few a-priori assumptions as possible.

\section{GENERAL DISCUSSION}

The concept of fixing ambiguities as a datum is identical to the concept of fixing one clock as a datum in a multi-station network solution. In the same way that all clocks are then estimated relative to that clock, so with the decoupled clock model all the phase clocks are computed with respect to a network datum clock plus an ambiguity datum. Apart from an arbitrary bias, the formal nature of the phase clock estimates is unchanged relative to the standard model clock estimates. 
Since the decoupled clock model is a generic processing model, it can be applied directly to Precise Point Positioning with the advantage that it facilitates Ambiguity Resolution (PPP-AR). Just as for standard PPP, PPP-AR requires precise orbit and clock corrections for each observed satellite, but in this case each observable requires its own satellite clock parameter, namely a carrier phase clock, a pseudorange clock, and a widelane (clock-like) bias. The source of such corrections can only be a network solution using the decoupled clock model. Standard model clock corrections, such as those provided by the IGS, cannot facilitate PPP-AR because they contain constant code biases and do not contain the phase biases. At the same time however, because time synchronization alone is the key to undifferenced ambiguity resolution, regular orbit corrections can be used. In principle, such corrections and subsequent models must be accurate enough that the total measurement error is not greater than half the narrowlane wavelength.

Figure 4 describes the conceptual relationship between the network and single-receiver solutions, where external precise orbits are used in both solutions and only clock errors are estimated for the satellites in the network solution. Due to the relative stability of the satellite code biases, the provision of the full-set of satellite clock corrections for PPP can be simplified by providing the pseudorange minus carrier phase clock difference. For real-time correction distribution, this is likely to be the optimum method to minimize the bandwidth required to deliver corrections to a user.

From a philosophical point of view, the decoupled clock model conforms to the original principles of PPP, and in fact GPS, whereby purely undifferenced observables are used, and all 


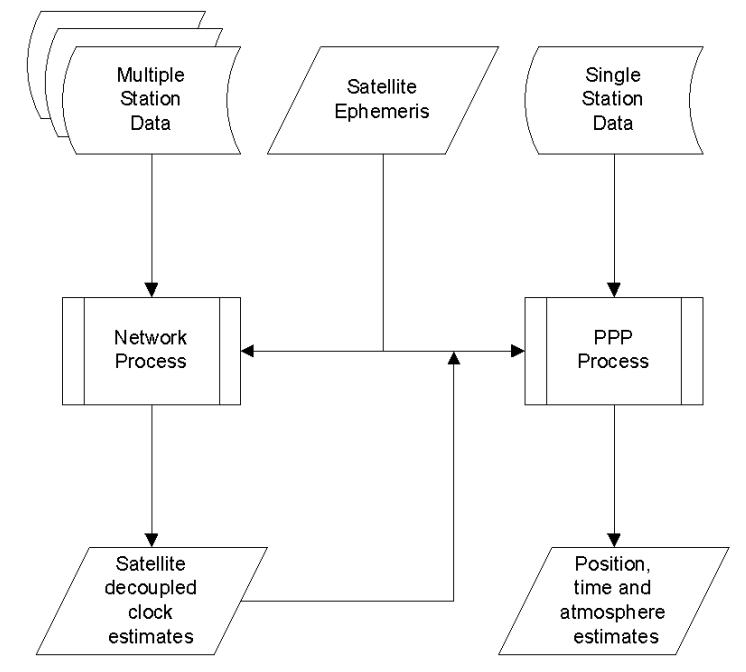

Figure 4. Schematic Network and PPP Processing Overview. the user-required information is contained in satellite-only corrections. More practically, it is stressed that the decoupled clock model represents a significantly more rigorous model of the GPS observations, and, even without explicit ambiguity resolution, should provide more consistent solutions over those provided by the standard undifferenced observation model.

The principle of the decoupled clock model can be extended to the processing of measurements from other, and multiple, Global Navigation Satellite Systems. The requirement for observationdependent clock parameters is determined by the differing signal structure of each system and the consequent hardware and software implementations of both the transmitters and the receivers [8]. Where these biases are stable with known values (e.g., for the GPS satellites), it is straightforward to correct the necessary observables to match the reference set [7]. In a similar manner, any carrier phase quadrature bias can also be corrected for. 


\section{NETWORK PROCESSING}

In the network solution, explicit ambiguity resolution and fixing is performed to minimize the number of normal equations and the size of the corresponding coefficient matrix. A simplified 2D LAMBDA transformation is applied to each widelane and ionosphere-free combination pair to help reduce the inherent correlation and speed-up convergence. A decision to fix is made when both the formal sigma and the float ambiguity difference from an integer drop below a common threshold. A value of 0.15 cycles was found to be suitable. In practice it is only possible to fix the amplified iono-free ambiguities after the corresponding widelane ambiguity has been fixed. In general, after an initial period of 30 minutes or so, $85 \%$ of ambiguities are fixed for the remainder of solution. The number of fixed ambiguities is not constant due to satellites rising and setting. Most of the unfixed ambiguities belong to rising satellites.

Figure 5 and Figure 6 present examples of station and satellite clock errors from a fixed ambiguity network solution. Figure 5 presents the phase-clock, code-clock difference and widelane bias for station YELL as previously presented in Figure 1. The IGS Rapid solution is also plotted for comparison. The widelane bias is clearly not stable over this period, exhibiting approximately a 3ns range over the 7-day period. Figure 6 presents the same parameters for PRN21/SVN45. For this satellite, as for the others, the code-phase clock difference and the widelane bias are noisy but largely constant. The 'bow-tie' effect in the code-phase differences is due to the influence of station low-elevation multipath. 


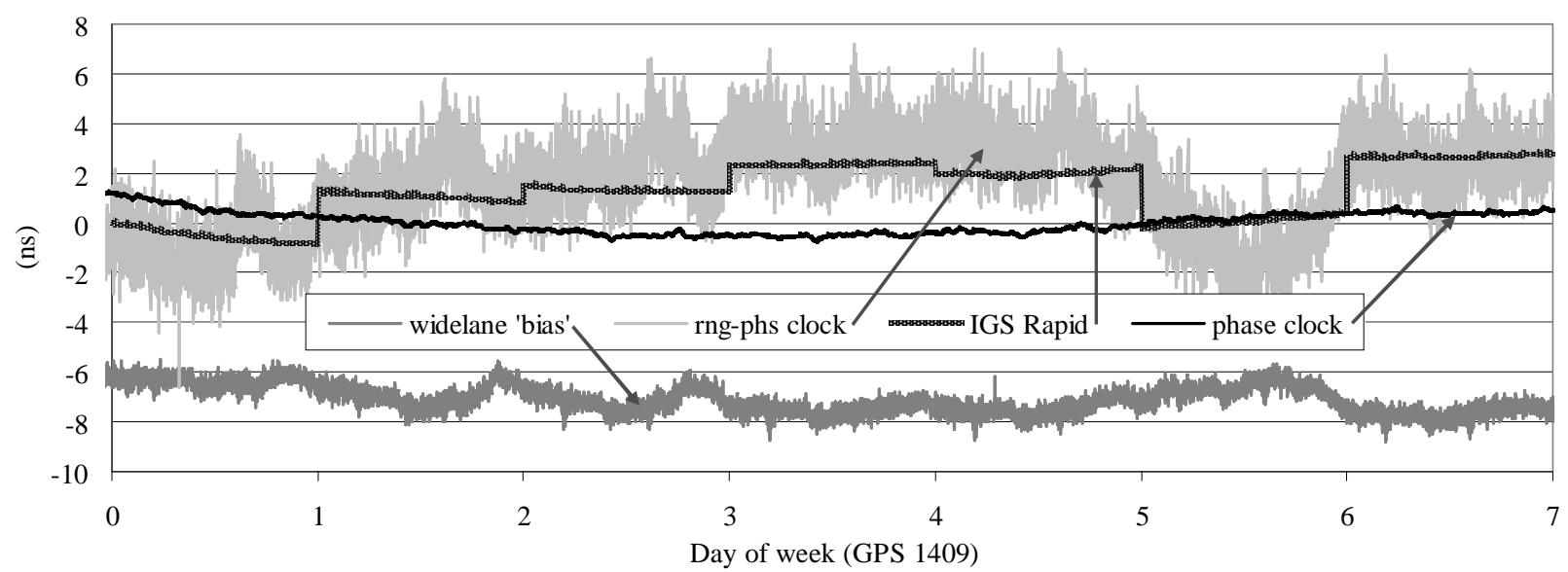

Figure 5. Yellowknife phase clock, code-phase clock difference and widelane bias estimates.

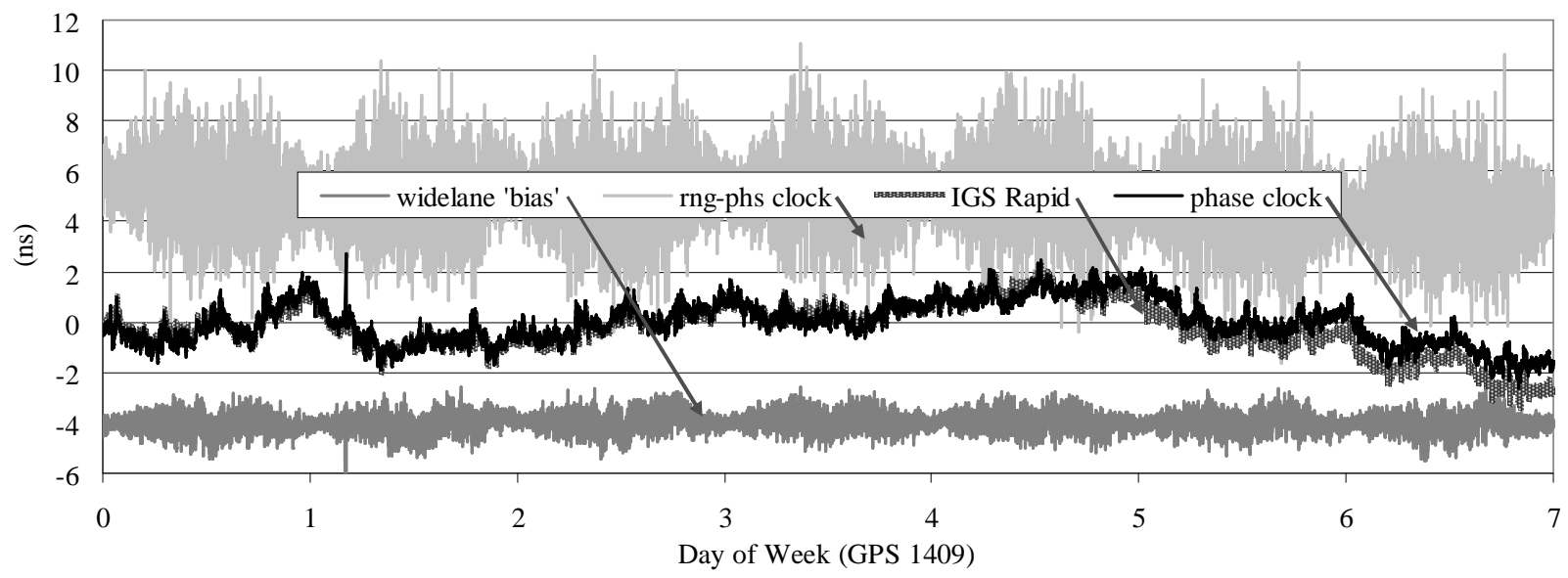

Figure 6. PRN21 phase clock, code-phase clock difference and widelane bias estimates.

Several satellite clocks experience a small drift relative to the Rapid solution similar to that evident in the last few days of Figure 6. Whether this is due to the drift of the Rapid solution or an artifact of incorrect ambiguity fixing is unclear. The IGS Rapid satellite clocks experience significantly smaller day-boundary jumps than the station clocks, making detection of a day-today drift more difficult. Regardless, the overall stability exhibited by the satellite code clock and widelane bias is clear, with the implication that a-posteriori smoothing could be applied for the purpose of providing slow-update corrections for real-time PPP users. The important point is 
that an assumption of bias constancy does not have to be made a-priori when using the decoupled clock model.

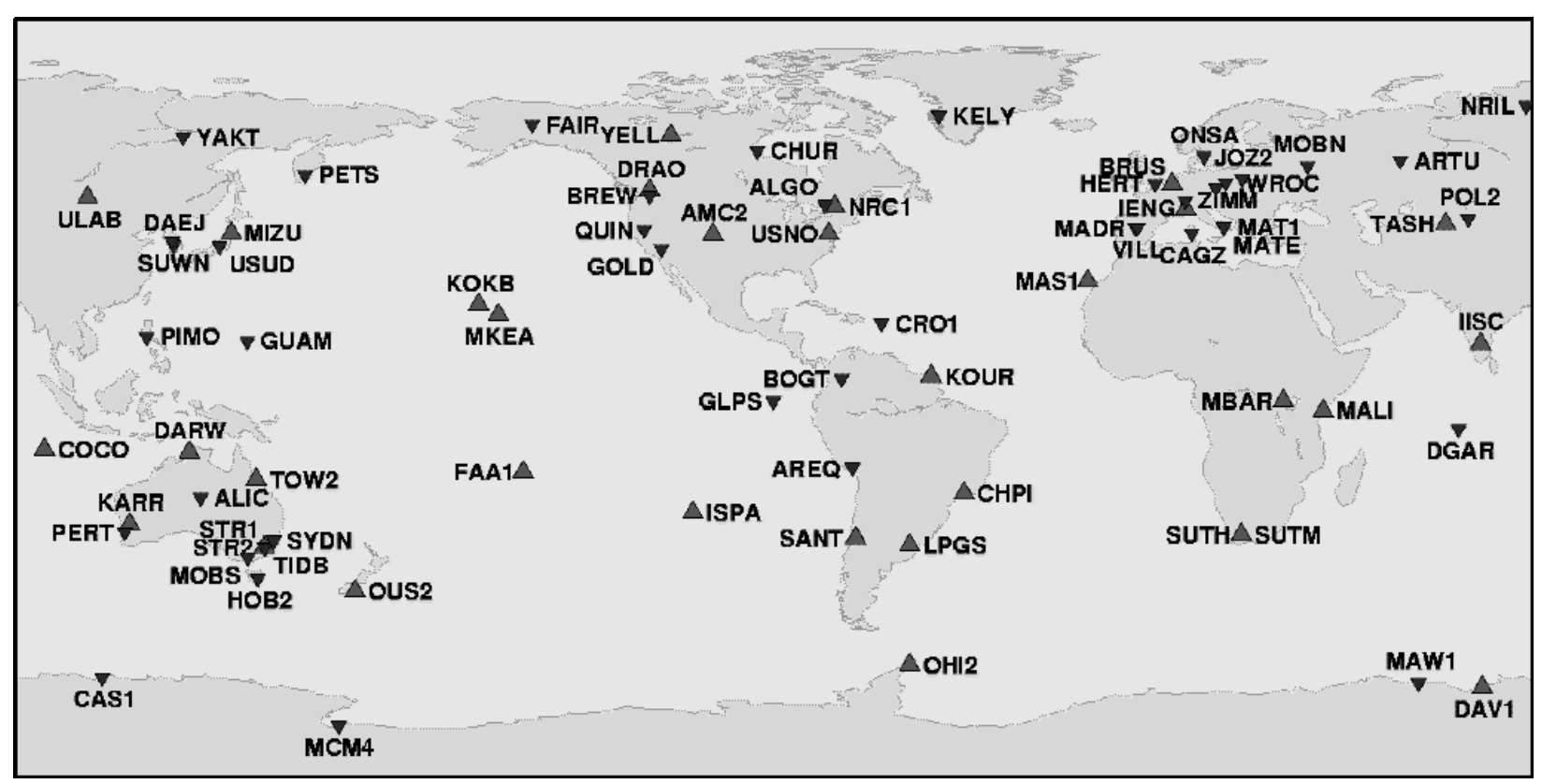

Figure 7. Network $(\Delta)$ and PPP testing stations ( $\nabla)$.

The network processed to support the PPP results in this paper is depicted in Figure 7. Thirtytwo IGS stations providing both L1 and L2 P(Y) code observations (P1 and P2) were selected for the 7 days corresponding to GPS week 1409 (January $7^{\text {th }}$ to January $13^{\text {th }}, 2007$ ). The satellite orbits were fixed to the IGS Rapid Product and the station coordinates were fixed to the weekly SINEX values [http://igscb.jpl.nasa.gov/components/prods.html]. The estimated parameters comprised white noise station and satellite clock errors plus a random-walk tropospheric zenith delay per station. The full data rate of 30 seconds was used. The number of stations required for the global decoupled clock solution is no more or less than for the standard model solution. 
The data was processed in a sequential least-squares filter (no smoothing) to provide a set of standard-model clocks, comparable to the IGS clocks, and a set of decoupled-model clocks. Explicit, in-solution, ambiguity fixing was applied to derive the decoupled clocks. Table 1 shows the daily RMS error of the standard satellite clocks compared to the IGS Rapid clocks as well as both the standard and decoupled daily repeatability (constellation epoch average removed) relative to the IGS Rapid clocks. No RMS error was computed for the decoupled clocks because of their ambiguous nature. However, it is clear from the repeatability that the decoupled clocks show an average error of $\sim 1 \mathrm{~cm}$, a small improvement over the standard clocks.

Table 1. Standard and decoupled satellite phase clock daily RMS error and repeatability $(\mathrm{cm})$.

\begin{tabular}{|l|l|c|c|}
\hline \multirow{2}{*}{ day } & \multicolumn{2}{|c|}{ Standard } & Decoupled \\
\cline { 2 - 4 } & RMS & repeatability & repeatability \\
\hline 07 & 4.6 & 2.0 & 1.0 \\
\hline 08 & 7.3 & 3.0 & 0.9 \\
\hline 09 & 5.7 & 1.8 & 0.9 \\
\hline 10 & 5.7 & 1.1 & 0.9 \\
\hline 11 & 5.6 & 1.3 & 1.0 \\
\hline 12 & 6.9 & 1.9 & 1.2 \\
\hline 13 & 7.2 & 2.3 & 1.1 \\
\hline AVG & $\mathbf{6 . 1}$ & $\mathbf{1 . 9}$ & $\mathbf{1 . 0}$ \\
\hline
\end{tabular}

\section{PPP PROCESSING}

The stations selected for testing PPP with ambiguity resolution are also depicted in Figure 7. None of these stations were used in the network processing to derive the decoupled satellite clocks. Of the 44 PPP stations in total, in general only $\sim 40$ are available at any one time due to variable data availability. Ten of the stations provided no L1 P-code (P1) observations. When 
processing these stations, the L1 C/A-code (C1) observations were corrected to P1 using the IGS P1-C1 satellite biases [7].

For each PPP run, the satellite clock and orbit parameters were held fixed. The station coordinates were estimated as unknown static values (no a-priori weight), the station clock errors (phase, range, widelane) were estimated as white-noise, and the tropospheric zenith delay was estimated as a random walk process. The elevation mask angle was set to 7.5 degrees, the recommended value for the Global Mapping Functions (GMF) [3], which were used to map the tropospheric zenith delays and to form the measurement model partial derivatives. One satellite's ambiguities are fixed a-priori as the datum ambiguities using rounded pseudorange measurements as estimates. A rigorous transformation is performed if a new satellite is required as a datum (due to a cycle slip, or setting below the elevation mask, etc.).

Ambiguity resolution was performed using the LAMBDA technique [21] on an epoch-by-epoch basis. That is to say, the underlying float solution was maintained independently with the ambiguity-fixed solutions computed separately (see Figure 8). This is an optimum way of combining recursive filtering with integer state estimation [9]. It is also a useful way of maintaining a float solution for comparison purposes. The main benefit of this approach is that the float solution remains uncorrupted by possibly incorrect ambiguity fixes. A disadvantage is that the solution can diverge should an undetected cycle-slip occur. Also, the underlying solution is somewhat weaker because the ambiguities of setting satellites are rigorously eliminated (i.e., retained implicitly) instead of being fixed (i.e., removed explicitly). 


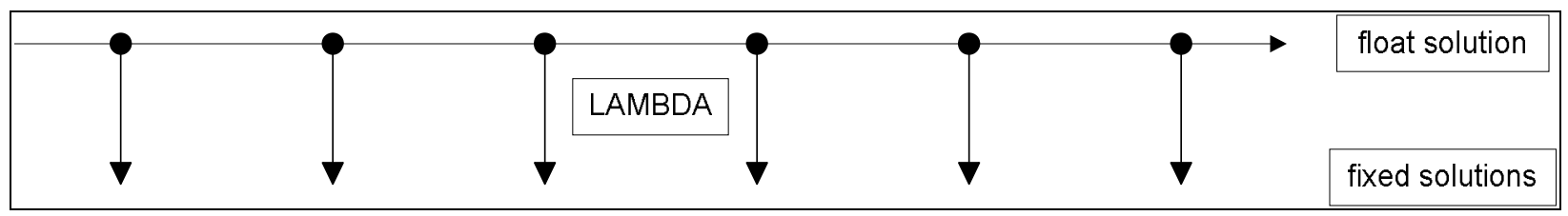

Figure 8. Schematic representation of PPP-AR processing.

When double-difference ambiguity resolution is undertaken, the general practice followed is to validate the ambiguities before explicit fixing. This was briefly investigated for the undifferenced ambiguity resolution performed here, but it was not found possible to meaningfully correlate any of the typical validation statistics with the actual positioning results obtained. One possible reason for this could be that the observation weight matrix used in our processing was optimistic, because it ignored the correlations between the three observables. It should be pointed out however, that the typical validation tests are not rigorously defined and have high false rejection rates $[25,26]$. Therefore, for this paper, the usefulness of ambiguity resolution with PPP was determined by examining the actual positioning results obtained with the technique. Reference coordinates for these comparisons were taken from the corresponding weekly SINEX file produced by the IGS.

\section{PPP RESULTS}

The initial PPP processing consisted of computing 5-minute solutions of daily files. The final daily latitude, longitude and height RMS errors for the total 7 day, 44 station data set are given in Table 2. What is immediately obvious is that there is only a small improvement, if any, provided by PPP-AR over standard PPP with 24 hours of data. There is an improvement in easting 
(longitude) from $0.8 \mathrm{~cm}$ to $0.4 \mathrm{~cm}$ which, though small, is consistent with what has been noted previously with double-difference ambiguity resolution [e.g., 2, 12]. The height component is not improved at all.

Table 2 also indicates that there is a small improvement with the decoupled clock model float solution over the standard model float solution. This is most likely due to the better parameterization of the code biases. These are properly accounted for in the decoupled clock model, but are dispersed among the parameters and residuals of the standard Table 2. Daily RMS static position errors for standard (STD) and decoupled (DCL) model. Given the only marginal improvement over the standard model, the decoupled clock model float solution will be used to compare with the ambiguity-fixed solution.

\begin{tabular}{|l|l|l|l|}
\hline RMS (cm) & Lat. & Lon. & Hgt. \\
\hline STD & 0.6 & 0.8 & 1.4 \\
\hline DCL-float & 0.4 & 0.6 & 1.4 \\
\hline DCL-fixed & 0.5 & 0.4 & 1.4 \\
\hline
\end{tabular}

Figure 9 shows a typical daily run with ambiguity resolution for station BOGT. Examining this figure shows three common attributes observed with this data set: i) the float-latitude component converges quickly; ii) the float longitude converges slowly; and iii) the height component is the weakest overall. There are two important consequences of AR that should be recognized, one good and one not. On the positive side, the horizontal components of position both converged very quickly, but on the negative side, as the height component shows, any underlying bias or weakness in the solution cannot be overcome with ambiguity fixing. The latter is practical confirmation of the expected performance of ambiguity fixed solutions as discussed by [23]. 


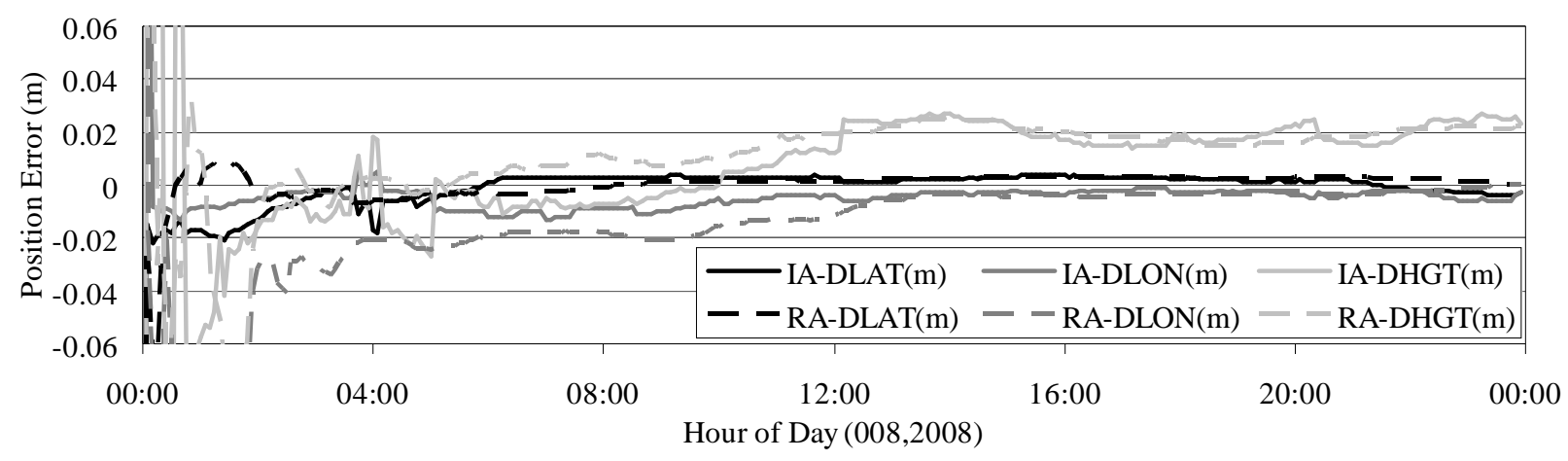

Figure 9. Station BOGT 24-hour static positioning at 5 minute rate for $8^{\text {th }}$ January 2008. Decoupled Clock Model real ambiguity (RA) float solutions and integer ambiguity (IA) fixed solutions.

To examine more closely the impact of AR on position convergence, the entire PPP data set was broken down into hourly files. The net result was approximately 7000 solutions from the 44 stations. This is less than the expected number, but some hours were missing and each hour was required to have $90 \%$ of epochs available to be processed. These were then processed with the same parameter set-up as the daily files, but at a 30 second data rate.

As an example of the overall results, the epoch average of the hourly float and fixed solutions with corresponding 1-sgima error bars are plotted for one station's horizontal and vertical errors in Figure 10 and Figure 11. In general, the horizontal error reaches the centimeter level, but this is only obvious after all 24 runs achieve convergence after 31 minutes, when the sigma is drastically reduced. In the vertical component, the same lack of significant improvement as the daily solutions is evident, but there is some small improvement in both the average and the spread of the results after the last solution has converged. 


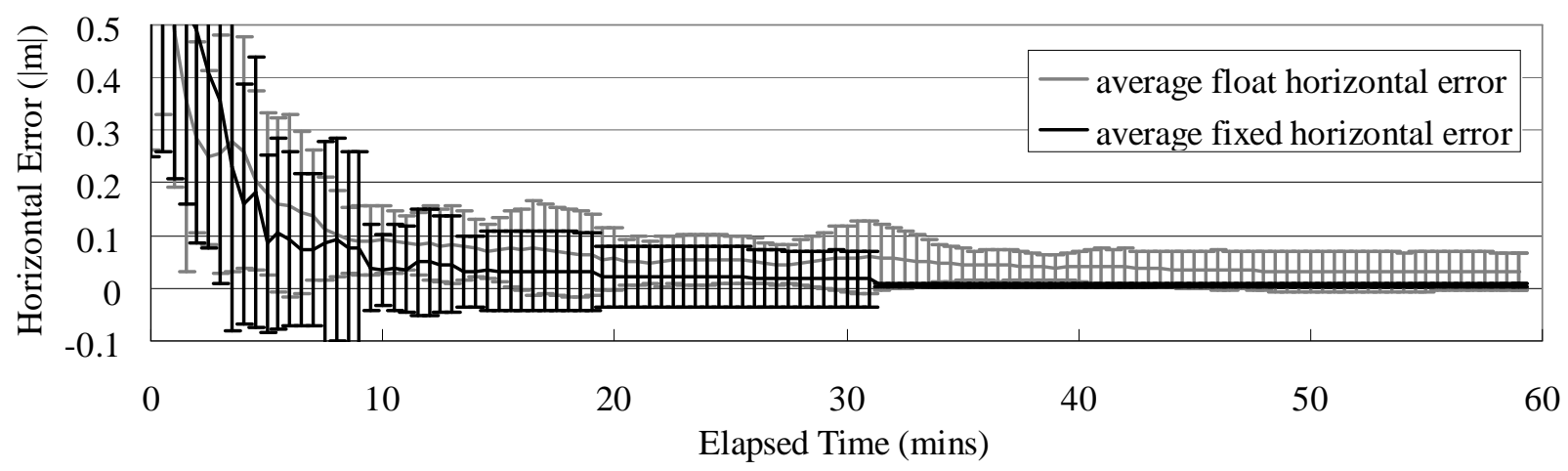

Figure 10. Average horizontal float (grey) and fixed (black) position error for station ALGO, $7^{\text {th }}$ January 2007 (error bars are 1 $\sigma$ ).

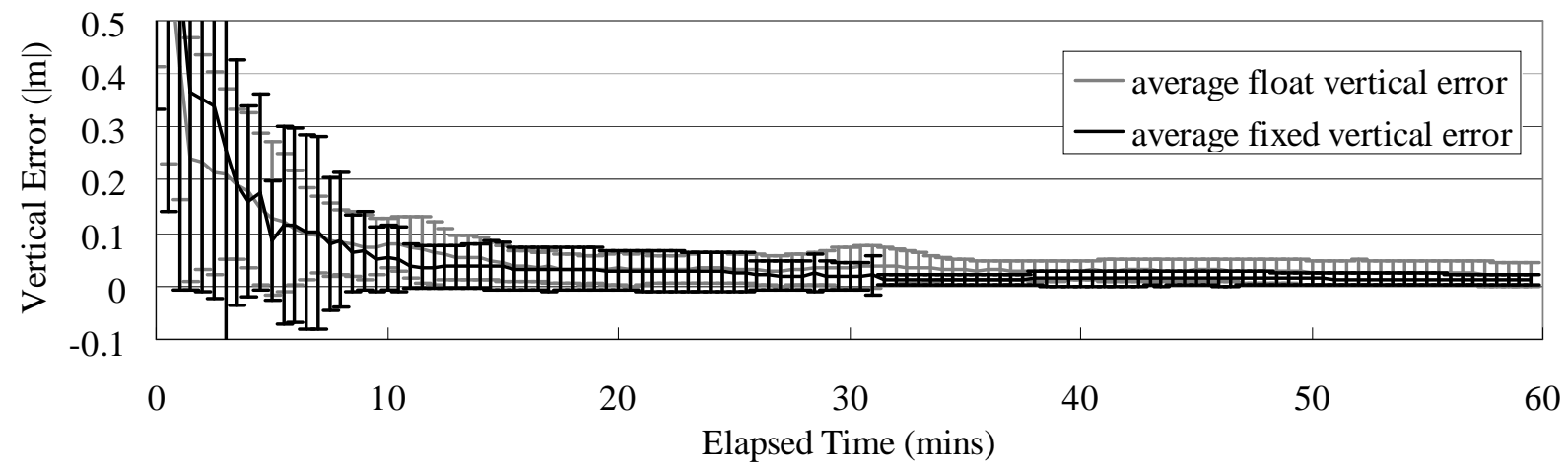

Figure 11. Average vertical float (grey) and fixed (black) position error for station ALGO, $7^{\text {th }}$ January 2007 (error bars are 1 $\sigma$ ).

Because individual solutions that converge slowly can skew the dispersion of the fixed position errors, the entire data set is portrayed in Figure 12 and Figure 13 using error percentiles. Figure 12 indicates that $90 \%$ of the float horizontal solutions are at the $10 \mathrm{~cm}$ level or better after 60 minutes. With ambiguity resolution, that level is reached in only approximately 30 minutes. At 60 minutes, $90 \%$ of the ambiguity-fixed results are at the $2 \mathrm{~cm}$ error level. A small number of solutions $(<10 \%)$ converge to sub-centimeter level in several minutes. The generally small improvement in height is reflected in Figure 13. In both plots it is noticeable that the fixed-error curve is above the float-error curve at the start of processing. This difference indicates that 
attempting ambiguity fixing immediately provides some position solutions that are slightly (but not significantly) worse than the float solution.

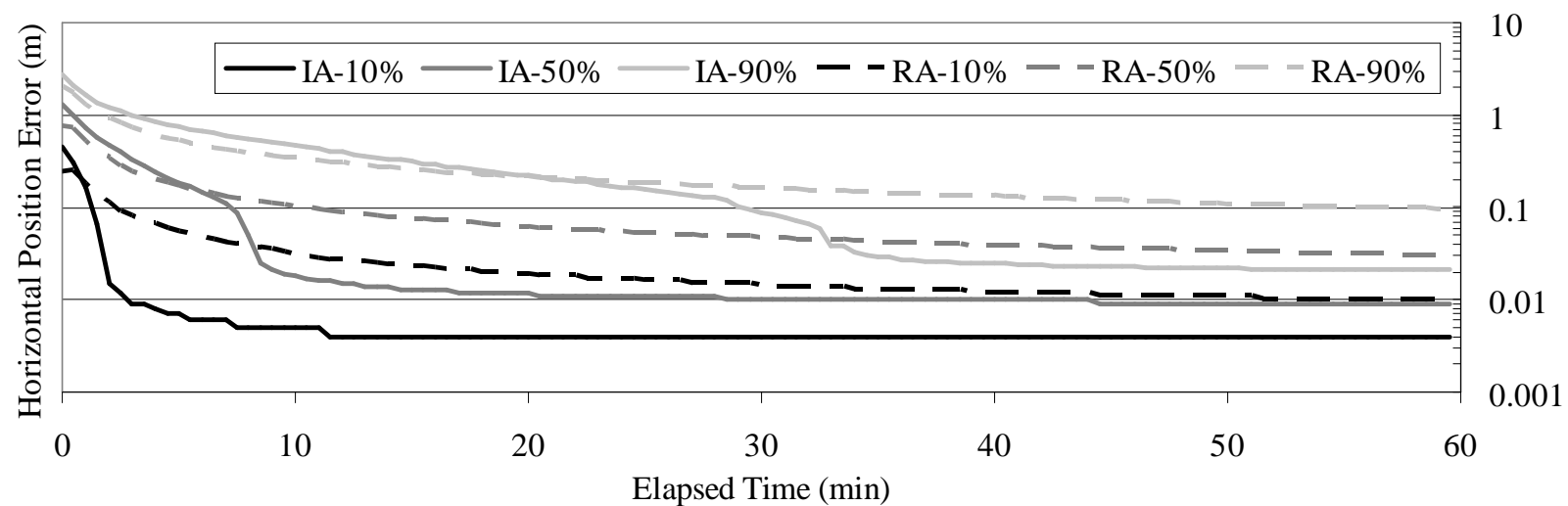

Figure 12. Horizontal real ambiguity (RA) and integer ambiguity (IA) position error 10, 50 and 90 percentiles (all data).

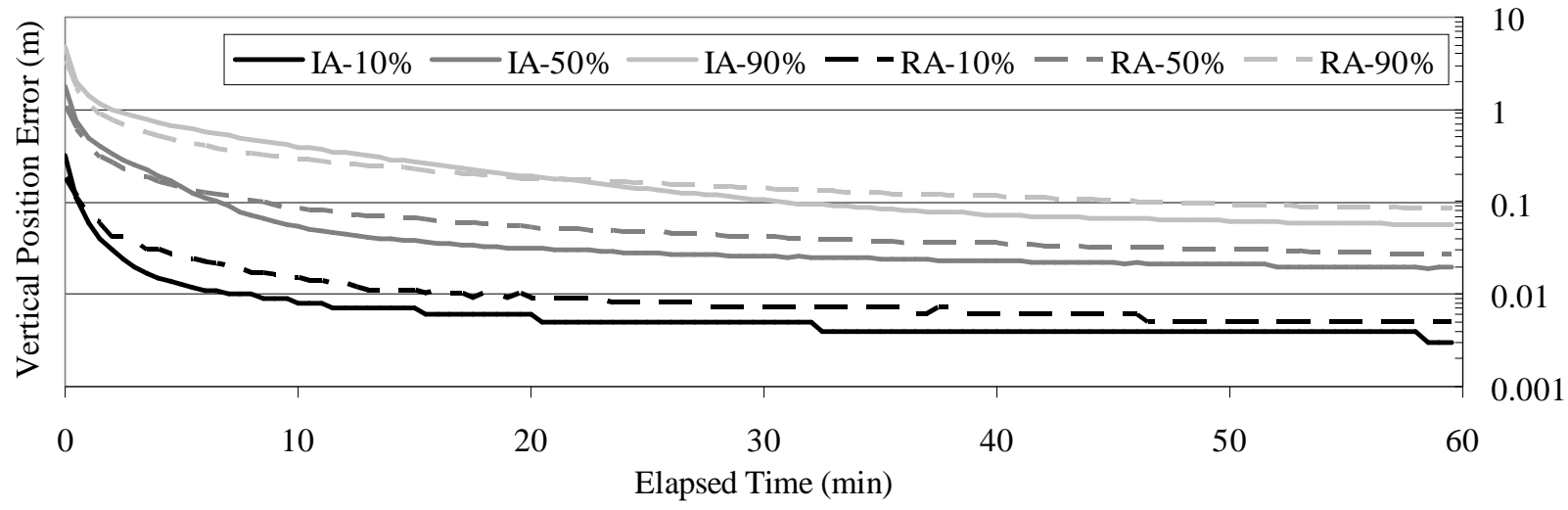

Figure 13. Vertical real ambiguity (RA) and integer ambiguity (IA) position error 10, 50 and 90 percentiles (all data).

The results in Figure 12 and Figure 13 represent all the data processed. It is not uncommon for some stations to experience divergence at some point in the data set, due to what appears to be a combination of undetected cycle slips, complete loss of lock, relatively low satellite count, poor geometry and poor quality pseudorange. A suitable validation scheme would hopefully screen these solutions, and this will be the subject of future work. 
To emphasize the impact of ambiguity resolution on position accuracy, Figure 12 and Figure 13 are summarized by Figure 14 and Figure 15, respectively. These plots represent the relative frequency of the horizontal and vertical errors after 60 minutes of processing. They can be considered as 'end-on' views of Figure 14 and Figure 15, respectively. It is especially clear from the distribution of horizontal errors that ambiguity resolution provides a more 'peaked' distribution, in addition to shifting the general mass of the distribution downward. This is also true for the vertical errors, but to a much lesser degree.

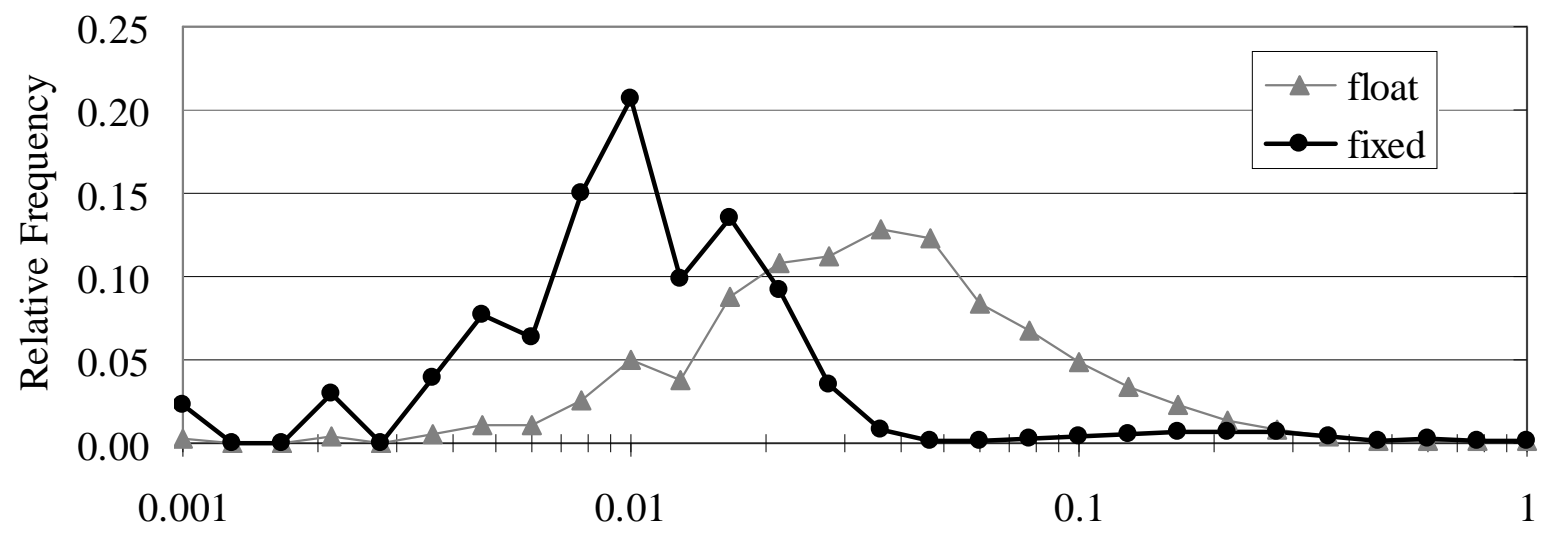

Horizontal Position Error (m)

Figure 14. Empirical horizontal error distributions for float and fixed ambiguity solutions after 60 minutes convergence time.

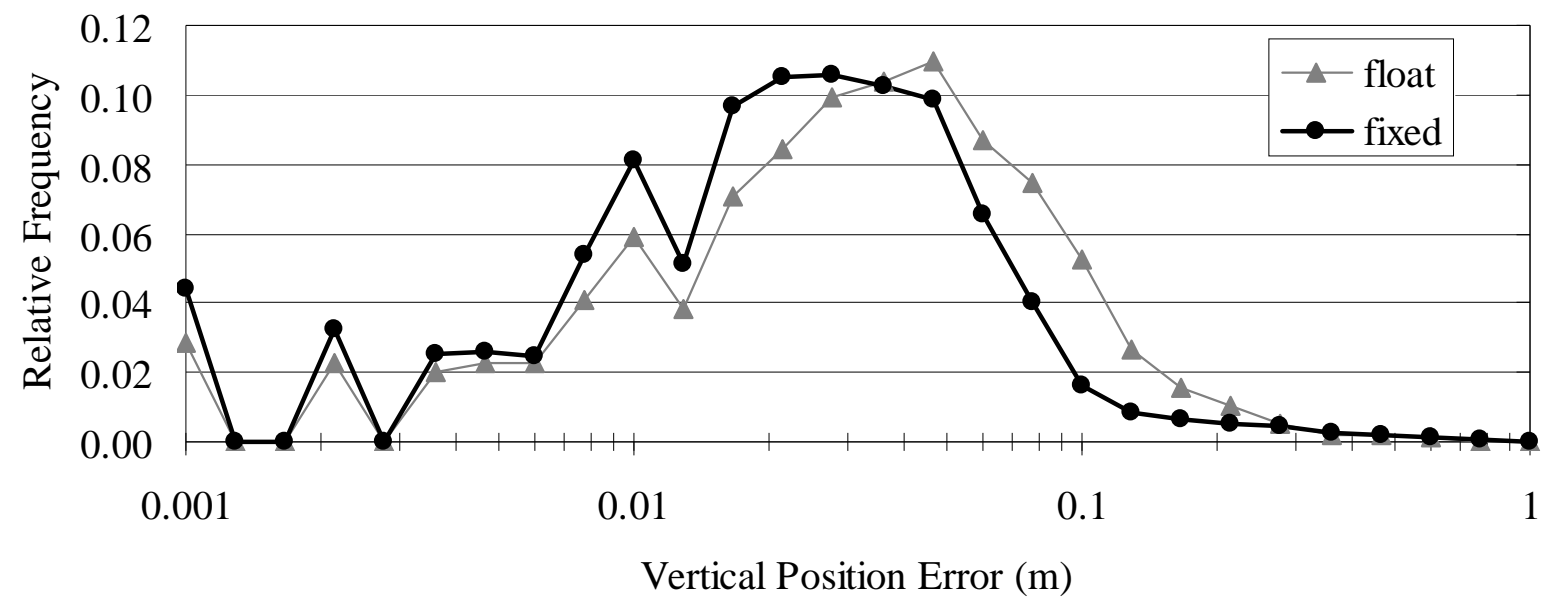

Figure 15. Empirical vertical error distributions for float and fixed ambiguity solutions after 60 minutes convergence time. 


\section{CONCLUSIONS}

This paper has sought to show that the issue of rigorously isolating the ambiguities of undifferenced GPS carrier-phases is primarily related to the corresponding isolation of the codebiases. The standard dual-frequency observation model combining ionosphere-free code and phase measurements cannot facilitate the explicit isolation of integer ambiguities because of the subtle role played by the code biases. The clock parameters, code and phase biases and ambiguities are inherently correlated with each other, and therefore combine to destructively interfere with each other. Fortunately, by decoupling the apparently distinct code and phase oscillator parameters, the effect of the code biases can be removed from the ambiguities and these can then be explicitly isolated as integer valued parameters by explicitly fixing a subset to arbitrary integer values.

This model and process is referred to as the decoupled clock model with ambiguity datum fixing, and isolates undifferenced carrier-phase ambiguities as integer-valued parameters. The primary consequence of this model is that Precise Point Positioning with Ambiguity Resolution (PPPAR) becomes possible. A network solution is required to compute the satellite clock corrections for each observable. These clock corrections contain all the necessary information to isolate the ambiguities as integers for single-station users. In this way, AR methods such as LAMBDA can be directly applied to PPP. With a 24-hour data set, ambiguity resolution makes only a small improvement over standard static PPP, making the longitude error generally comparable to the latitude error. PPP-AR does however accelerate the overall solution convergence to give $\mathrm{cm}$ level horizontal accuracy after 1 hour or less. Several of the stations processed here showed 
horizontal convergence in several minutes. Improvement in vertical convergence was less significant and most likely limited by the inherent geometric weakness compared to the horizontal components of position.

More work is required to investigate the impact of kinematic data, overall data quality, satellite geometry and robust ambiguity validation, but PPP-AR with the decoupled clock model has the capability to reduce the observation time required for precise positioning. One benefit of the decoupled clock model compared to other recently introduced undifferenced ambiguity techniques, is that no a-priori assumption about the stability of code biases is required for either the satellite transmitters or the user receivers. Provided that the code, phase and widelane clock and bias parameters are estimated as white-noise processes, they will entirely accommodate the common-channel timing characteristics of the observables. From a philosophical point of view, the decoupled clock model conforms to the original principles of PPP and GPS, whereby purely undifferenced observables are used, and all the user-required information is contained in satellite-only corrections. From a practical point of view, this may permit currently operating PPP correction services to provide decoupled satellite clocks by extending their current message set to broadcast the additional corrections.

\section{ACKNOWLEDGMENTS}

The authors wish to acknowledge the support of their colleagues at the Geodetic Survey Division, at York University, and in the wider Canadian geodetic academic community. The 
results presented in this paper are derived from data and products provided by Natural Resources Canada and the International GNSS Service. 


\section{REFERENCES}

1. Bisnath, S. and Y. Gao (2009). "Precise Point Positioning: A Powerful Technique with a Promising Future." GPS World, April, pp.43-50.

2. Blewitt, G. (1989). "Carrier Phase Ambiguity Resolution for the Global Positioning System Applied to Geodetic Baselines up to $2000 \mathrm{~km}$." Journal of Geophysical Research, Vol. 94, No. B8, pp. 10,187-10,203.

3. Boehm, J., A. Niell, P. Tregoning, and H. Schuh (2006). "Global Mapping Function (GMF): A new empirical mapping function based on numerical weather model data." Geophysical Research Letters, Vol. 33, paper L07304.

4. Bossler, J.D., C.C. Goad and P.L. Bender (1980). "Using the Global Positioning System (GPS) for Geodetic Positioning." Bulletin Géodesique, Vol. 54, pp. 553-563.

5. Collins, P. (2008). "Isolating and Estimating Undifferenced GPS Integer Ambiguities." Proceedings of ION-NTM-2008, San Diego, Calif., 28-30 January, pp. 720-732.

6. Collins, P., F. Lahaye, P. Héroux and S. Bisnath (2008). "Precise Point Positioning with Ambiguity Resolution using the Decoupled Clock Model." Proceedings of ION-GNSS-2008, Savannah, Georgia, 16-19 September, pp. 1315-1322.

7. Collins, P., Y. Gao, F. Lahaye, P. Héroux, K. MacLeod and K. Chen (2005). "Accessing and Processing Real-Time GPS Corrections for Precise Point Positioning - Some User Considerations" Proceedings of ION-GNSS-2005, Long Beach, Calif., 13-16 September, pp. 1483-1491.

8. Collins, P. and S. Bisnath (2009). "Characterization of Hardware Biases in Global Positioning System Measurements" In preparation.

9. Cox, D.B. and J.D.W. Brading (2002). "An Optimum Recursive Filter with Integer States (ORFIS) for Relative Navigation with GPS Accumulated-Phase Measurements." Proceedings of ION-GPS-2002, Portland, Ore., 24-27 September, pp. 2768-2773.

10. Dach, R., U. Hugentobler, T. Schildknecht, L-G. Bernier and G. Dudle (2005). "Precise Continuous Time and Frequency Transfer Using GPS Carrier Phase." Proceedings of the IEEE International Frequency Control Symposium and Exposition, Vancouver, B.C., 29-31 August.

11. Defraigne, P. and C. Bruyninx (2007). "On the link between GPS pseudorange noise and day-boundary discontinuities in geodetic time transfer solutions." GPS Solutions, Vol. 11, pp. 239-249. 
12. Dong, D. and Y. Bock (1989). "Global Positioning System Network Analysis With Phase Ambiguity Resolution Applied to Crustal Deformation Studies in California." Journal of Geophysical Research, Vol. 94, No. B8, pp. 3949-3966.

13. Ge, M., G. Gendt, M. Rothacher, C. Shi and J. Liu (2008). "Resolution of GPS carrier-phase ambiguities in Precise Point Positioning (PPP) with daily observations." Journal of Geodesy, Vol. 82, pp.389-399.

14. Goad, C.C. (1985). "Precise Relative Position Determination Using Global Positioning System Carrier Phase Measurements in a Nondifference Mode." Proceedings of the First International Symposium on Precise Positioning with the Global Positioning System, US Dept. of Commerce, Rockville, Maryland, 15-19 April, Vol. 1, pp. 347-356.

15. Guyennon, N., G. Cerretto, P. Tavella and F. Lahaye (2009). "Further Characterization of the Time Transfer Capabilities of Precise Point Positioning (PPP): The Sliding Batch Procedure." IEEE Transactions on Ultrasonics, Ferroelectrics, and Frequency Control, Vol. 56, No. 8, pp. 1634-1641.

16. Héroux, P. and J. Kouba (2001). "GPS Precise Point Positioning Using IGS Orbit Products." Physics and Chemistry of the Earth (A), Vol. 26, No. 6-8, pp. 572-578.

17. Laurichesse, D. and F. Mercier (2007). "Integer ambiguity resolution on undifferenced GPS phase measurements and its application to PPP." Proceedings of ION-GNSS-2007, Fort Worth, Texas, 25-28 September, pp. 839-848.

18. Laurichesse, D., F. Mercier, J.P. Berthias, P. Broca and L. Cerri (2009). "Integer Ambiguity Resolution on Undifferenced GPS Phase Measurements and Its Application to PPP and Satellite Precise Orbit Determination." Navigation: Journal of the Institute of Navigation, Vol. 56, No. 2, Summer, pp.135-149.

19. Ray, J., and K. Senior (2002) "IGS/BIPM Pilot Project: GPS carrier phase for time/frequency transfer and time scale formation", Metrologia, 40(3), S270-S288, 2003; erratum, 40(4), 205, 2003.

20. Remondi, B.W. (1985). "Global Positioning System Carrier Phase: Description and Use." Bulletin Géodesique, Vol. 59, pp. 361-377.

21. Teunissen, P.J.G. (1994). "A New Method for Fast Carrier Phase Ambiguity Estimation." Proceedings of PLANS'94: IEEE Position, Location and Navigation Symposium, Las Vegas, Nevada, 11-15 April, pp. 562-573.

22. Teunissen, P.J.G. (1996). "Rank defect integer least-squares with applications to GPS." Bollettino di Geodesia e Scienze Affini, No. 3, pp. 225-238

23. Teunissen, P.J.G. (1999). "The Mean and the Variance Matrix of the 'Fixed' GPS Baseline." Acta Geodaetica et Geophysica Hungarica. Vol. 34, No. 1-2, pp. 33-40. 
24. Teunissen, P.J.G. and D. Odijk (2003). "Rank-defect integer estimation and phase-only modernized GPS ambiguity resolution." Journal of Geodesy, Vol. 76, pp. 523-535.

25. Verhagen, S. (2004). "Integer ambiguity validation: An open problem?" GPS Solutions, Vol. 8, pp. 36-43.

26. Verhagen, S. (2005). "On the Reliability of Integer Ambiguity Resolution." Navigation: Journal of The Institute of Navigation, Vol. 52, No. 2, pp. 99-110.

27. Wells, D.E., W. Lindlohr, B. Schaffrin and E. Grafarend (1987). GPS Design: Undifferenced Carrier Beat Phase Observations and the Fundamental Differencing Theorem. Department of Geodesy and Geomatics Engineering Technical Report No. 116, University of New Brunswick, Fredericton, New Brunswick, Canada, 141 pp.

28. Zumberge, J.F., M.B. Heflin, D.C. Jefferson, M.M. Watkins and F.H. Webb (1997). "Precise point positioning for the efficient and robust analysis of GPS data from large networks." Journal of Geophysical Research, Vol. 102, No. B3, pp. 5005-5018. 